\title{
Burst Occipital Nerve Stimulation for Chronic Migraine and
}

\section{Chronic Cluster Headache}

Rodrigo Garcia-Ortega $\mathrm{RN}^{1}$, Tamara Edwards $\mathrm{RN}^{1}$, Liz Moir $\mathrm{RN}^{1}$, Tipu Z Aziz F.MedSci ${ }^{1,2,3}$, Alexander L Green FRCS(SN) ${ }^{1,2,3}$, James J FitzGerald PhD $D^{1,2,3}$

Institutional affiliations: ${ }^{1}$ Department of Neurosurgery, Oxford University Hospitals NHS Foundation Trust, Oxford, UK; ${ }^{2}$ Nuffield Department of Surgical Sciences, University of Oxford, Oxford, UK; ${ }^{3}$ Nuffield Department of Clinical Neurosciences, University of Oxford, Oxford, UK.

Sources of financial support: None

Corresponding author: James J FitzGerald, Department of Neurosurgery, Level 3, West Wing, John Radcliffe Hospital, Oxford, OX3 9DU, UK. Email james.fitzgerald@nds.ox.ac.uk 


\section{Abstract}

Background: Occipital nerve stimulation (ONS) is widely used for headache syndromes including chronic migraine $(\mathrm{CM})$ and chronic cluster headache $(\mathrm{CCH})$. The paraesthesia associated with tonic stimulation can be bothersome and can limit therapy. It is now clear in spinal cord stimulation that paraesthesiafree waveforms can produce effective analgesia, but this has not been reported in ONS for $\mathrm{CM}$ or $\mathrm{CCH}$.

Materials and Methods: 17 patients $(12 \mathrm{CM}$ and $5 \mathrm{CCH})$ were treated with bilateral burst pattern ONS, including 4 who had previously had tonic ONS. Results were assessed in terms of the frequency of headaches (number of headache days per month for $\mathrm{CM}$, and number of attacks per day for $\mathrm{CCH}$ ) and their intensity on the numeric pain rating scale.

Results: Burst ONS produced a statistically significant mean reduction of $\mathbf{1 0 . 2}$ headache days per month in $\mathrm{CM}$. In $\mathrm{CCH}$ there were significant mean reductions in headache frequency (92\%) and intensity (42\%).

Conclusions: Paraesthesia is not necessary for good quality analgesia in ONS. Larger studies will be required to determine whether the efficacies of the two stimulation modes differ. Burst ONS is imperceptible and therefore potentially amenable to robustly blinded clinical trials.

\section{Keywords}

Migraine; Cluster Headache; Occipital Nerve Stimulation; Neuromodulation 


\section{Introduction}

Occipital nerve stimulation (ONS) may be used to treat chronic severe headache that is resistant to medical therapy. ONS involves electrically stimulating the greater occipital nerves (GONs), which are the medial branches of the dorsal primary rami of C2. They emerge from deeper layers by piercing semispinalis capitis approximately $3 \mathrm{~cm}$ below and $1.5 \mathrm{~cm}$ lateral to the inion (1) and run upwards towards the vertex, supplying sensation to the posterior scalp. An ONS system consists of electrode wires that are inserted underneath the scalp to stimulate the GONs (usually one on each side, so as to be in a position to stimulate both left and right GONs), and an implanted pulse generator (IPG).

Migraine headache is usually one-sided, often throbbing, and may be accompanied by nausea/vomiting or sensitivity to light. Episodes last from hours up to a few days. The frequency of attacks is variable but patients who are considered for ONS have progressed to 'chronic migraine' (CM) where headache is present more days than not. Before considering ONS, patients will have tried preventative medication including beta blockers, topiramate, sodium valproate, pizotifen, amitriptyline, and gabapentin, and would usually also have tried Botulinum neurotoxin (BoNT) treatment.

In cluster headache, pain is located in or around the eye or temple and affects only one side of the head, which for most patients is always the same side. It is accompanied by other symptoms including facial flushing, tearing, and running nose. Individual attacks typically last from 15 minutes to 3 hours, and come in clusters lasting weeks to months during which there may be multiple episodes per day. In most cases there are lengthy headache-free periods (months to years) between clusters. However patients who are considered for invasive neuromodulation have 'chronic cluster headache' (CCH) where the long headache-free intervals between clusters are absent. This can be a progression from episodic cluster headache or it may arise de novo. Before considering neuromodulation, patients would usually have tried preventative medications including verapamil, steroids, lithium, topiramate and gabapentin. 
Neuromodulation options in chronic cluster headache include occipital nerve stimulation (ONS) (2-8), sphenopalatine ganglion (SPG) stimulation (9-11), and deep brain stimulation (DBS) (12-14) of the ipsilateral posterior hypothalamus. While all can be effective, ONS is the least invasive and is low risk, and is therefore our usual first line therapy.

ONS and DBS systems are 'always-on' and the aim of the treatment is preventive (in contrast to SPG stimulation which is used in an episodic manner in response to the onset of a cluster attack). ONS systems typically deliver a regular pattern of small current pulses to the nerves. This regular pattern is known as 'tonic' stimulation (which we will denote as tONS), and it generates a paraesthesia (tingling or buzzing sensation) in the scalp. As with tonic spinal cord stimulation (SCS), the traditional belief has been that this paraesthesia was a prerequisite for effective pain relief. However in SCS it is now clear that this is not the case, and spinal cord stimulator systems that cause no paraesthesia at all, yet give effective pain relief, are now widely available. Paraesthesia-free pain relief may be achieved by stimulating at much higher frequencies than usual (in the kiloHertz range rather than the typical $50 \mathrm{~Hz}$ ) $(15-17)$, or by delivering stimulation in bursts (short trains of pulses separated by a gap) $(18,19)$. Some studies have suggested that burst SCS may provide better pain relief than tonic stimulation and that most patients prefer not to have paraesthesia $(19,20)$.

In three large series of tONS for $\mathrm{CCH}$, attack frequencies were reduced by $46-58 \%$ (6-8). The response in migraine is relatively modest, with a reduction of less than 3 headache days per month when the control response is subtracted (21). There is clearly room for improvement in both conditions. Additionally, some patients dislike the scalp paraesthesia that accompanies tONS, which may limit therapy (7). In some cases patients have had to switch the system off at night to be able to sleep well (8). Therapy can also be limited by muscle activation. The successful use of paraesthesia-free burst pattern ONS (bONS) has recently been reported in two cases of occipital neuralgia (22), and the question therefore arises as 
to whether it might be effective in $\mathrm{CCH}$ and $\mathrm{CM}$. We present here the results of our first 17 cases treated with paraesthesia-free bONS.

\section{Materials and Methods}

\section{Patients}

All patients satisfied the current accepted definitions of $\mathrm{CM}$ or $\mathrm{CCH}$. $\mathrm{CM}$ is defined as headache present on at least 15 days per month for more than 3 months, with typical migraine features on at least 8 days per month, in the absence of medication overuse (23). $\mathrm{CCH}$ is defined as having cluster headache attacks for more than one year without remission or with remission lasting less than 3 months (23).

Operative procedure

All patients underwent implantation of their system in a single stage under general anaesthesia. With the patient in the lateral position, electrodes were placed bilaterally via a small vertical midline incision immediately below the inion, with leads being passed laterally from the incision, deep to trapezius, in the direction of the external auditory meatus. Insertion was facilitated by first making a tract using a dissector and then passing an introducer sheath ("Epiducer", Abbott) along this tract. Lead tips were approximately 5-6 centimetres from the midline, with the most proximal contacts just lateral to the incision. Electrode siting was confirmed by fluoroscopy. Lamitrode S8 single column paddle leads (Abbott) were implanted, placed with the exposed contacts on their superficial surface. From the insertion point, leads were tunnelled to a left subclavicular pocket where the IPG was sited. The IPG was either a Proclaim primary cell (patients $\mathrm{M} 1, \mathrm{C} 1$, and $\mathrm{C} 2$ ) or Prodigy rechargeable (all others) device (Abbott).

Programming 
Patients were either day cases or had a single overnight postoperative stay. Their devices were switched on after allowing two weeks for their surgical wounds to heal.

Tonic stimulation was used initially to find the electrode combination that covered the occipital nerve sensory distribution as much as possible. This will vary from individual to individual depending on exact lead position and GON anatomy. We used either a bipolar or a tripolar lead configuration (+ - +). The stimulation was then switched into burst mode. De Ridder pattern burst stimulation was used (5 pulses per burst, each of width $1000 \mu$ s separated by $1000 \mu$ s intervals, and overall frequency of bursts 40 $\mathrm{Hz}$ ). The threshold for perception of the burst stimulation was found and the stimulation set at $50 \%$ of this level. Patients were provided with a programmer with which they could vary the stimulation amplitude, with the maximum set to the perception threshold.

\section{Pain evaluation}

For $\mathrm{CM}$ the number of headache days per month was recorded, together with the average intensity of the headaches as measured on the eleven point numeric pain rating scale (NPRS).

For $\mathrm{CCH}$, patients were asked to report cluster attack frequency, and the average intensity of the headaches on the NPRS.

Statistical methods

Given the small numbers the data could not be tested for normality and thus non-parametric statistics were used. For descriptive statistics we have given mean and range, and for comparisons a sign test was used. 


\section{Results}

17 patients were treated. Patient details and results are summarised in table $1 . \ln 4$ cases $(1 \mathrm{CM}$ and 3 $\mathrm{CCH}$ ) the patient had previously had tONS but required revision surgery due to lead displacement or infection.

In all cases stimulation was paraesthesia-free and there were no cases where muscle activation occurred.

$C M$

12 patients had bONS for CM, 3 male and 9 female, with a mean age at treatment of 46 years (range 25 to 73). Preoperatively the mean number of headache days per month was 26.5 (range 15 to 30 ). The headache was present every day in 8 cases. Postoperatively there was a mean reduction in the number of headache days per month of $\mathbf{1 0 . 2}$ days for the group as a whole ( $p=\mathbf{0 . 0 0 2}$, one tailed sign test). Responses were dichotomous: $\mathbf{4}$ patients had no reduction in headache days at all, while the other $\mathbf{8}$ (patients M1, M4, M7, M8, M9, M10, M11, and M12) had a mean reduction of 15 days.

Mean headache intensity preoperatively was 7.8/10. The mean improvement in headache NPRS was only $16 \%$, but again this was divided into 6 of 12 patients who had an improvement in headache intensity averaging 32\%, and 6 in whom it remained unchanged. 4 of the 6 patients whose headache intensity improved were also in the group who had a reduction in headache days, while the other 2 had no reduction in headache days. Thus of $12 \mathrm{CM}$ patients, 4 had a reduction in both headache days and headache intensity; $\mathbf{4}$ had a reduction in headache days but not headache intensity, 2 had a reduction in headache intensity but not headache days, and $\mathbf{2}$ had no improvement of either kind.

$\mathrm{CCH}$ 
5 patients had bONS for $\mathrm{CCH}$, all male, with a mean age of 38 years at treatment (range 25 to 60 ). Preoperatively the mean cluster attack frequency was 7.6 per day (range 2 to 20) and all patients rated the intensity of their headaches as $10 / 10$ on the NPRS. Postoperatively the attack frequency was reduced by at least $80 \%$ in all cases, with 3 cases having either complete remission (patient $\mathrm{C} 1$ ) or a reduction in frequency to less than one episode per week (patients C2 and C5). Mean attack frequency reduction was $92 \%$ ( $p=0.013$, one tailed sign test). There was a mean reduction of $42 \%$ in the intensity of residual headaches ( $p=0.023$, one tailed sign test).

Complications

Two patients (M4 and $\mathrm{C} 4$ ) required explantation due to infection. Both of these patients had responded well and the pain data presented are the last data prior to system removal. Patient M4 has since been reimplanted and her pain relief is the same as it was prior to system removal. Patient C4 is awaiting reimplantation. 


\section{Discussion}

Early experience suggests that paraesthesia-free ONS using a burst waveform may be an effective treatment for severe chronic cluster and chronic migraine headaches.

This study shares many of the usual limitations inherent in retrospective clinical case series. There is no control group, and patients were not systematically trialled with both stimulation modes. This series does not therefore provide a direct comparison between tONS and bONS. Further studies are needed to compare the relative efficacies of the two modes of stimulation.

The issue of the necessity or not of paraesthesia in ONS for CM was previously addressed by Slotty et al (24), who compared the effects of stimulation amplitudes above and below the threshold of perception in chronic migraine patients who had already had a successful tONS implant. Stimulating at subthreshold levels yielded an efficacy that was intermediate between no stimulation and suprathreshold stimulation (mean VAS was 8.45 with stimulation off, 5.65 with subthreshold stimulation, and 1.98 with suprathreshold stimulation). This clearly demonstrated that it is possible to have at least some effect without paraesthesia. It could not however exclude the possibility that paraesthesia was necessary for optimal effect. The lesser effect of subthreshold stimulation could have been simply due to the fact that the stimulator amplitude had to be set low to avoid generating paraesthesia, which might be expected to make it less effective, but it could also be because having the paraesthesia is important. In contrast, stimulation in the patients presented here was paraesthesia-free by virtue of the nature of its waveform, rather than by virtue of setting a low amplitude. The finding that paraesthesia free bONS can give results that appear to be as good as tONS, with almost complete remission in some cases of $\mathrm{CCH}$, strongly suggests that paraesthesia is not necessary for optimal ONS results. This accords with an early report of good results in occipital neuralgia (22). 
Tonic ONS in CM has been subjected to four randomised controlled trials (RCTs) (25-28) and there are additionally several published case series (29-33). The way the results of treatment have been evaluated has not been entirely consistent from study to study, complicating comparison, but three of the RCTs reported a similar measure of the number of days per month affected by prolonged moderate to severe headache $(25,27,28)$. Meta-analysis of these yielded a reduction of 2.59 days per month $(95 \% \mathrm{Cl} 0.91-$ 4.27) in the active treatment group compared to sham control (21). Given that by definition CM patients have a minimum of 15 headache days per month, and often many more, this is a modest effect size. In the group presented here the mean reduction in headache days was 10.2. Whilst this was statistically significant and at first sight appears to compare favourably with the tONS results above, the data are not normally distributed and the number of cases is small, so it is not possible to put a confidence interval on this result, and we had no control group for comparison. In the RCTs the control groups improved by some 2-4 days; the apparent 10 day improvement here is therefore very likely to be an overestimate. Larger studies including controls will be required to determine effect size more precisely.

A striking feature of the results presented here was that the CM patients appeared to clearly separate into two groups. 4 of 12 had no reduction in headache days at all, while 8 had a mean reduction of 15 days, although only 5 of them (42\%) had a response of $50 \%$ or more. 6 of $12 \mathrm{CM}$ patients reported reduction in the intensity of their headaches. Only 4 of these were in the group with reduced headache days, thus it appears that if there is a response to treatment, it can take any of three forms: a reduction in headache days, a reduction in intensity, or a combination of both. The only potential preoperative predictor of outcome we observed was the number of headache days per month at baseline. All of the patients who failed to improve in terms of headache days had reported preoperatively that they had headache every day (see figure 1, upper left graph). This may simply have occurred by chance, and the same pattern is not observed in the NPRS. If it is genuine, it may indicate that those with headache 
every day have a headache syndrome that is in some way different and more refractory to ONS than those with fewer headache days; or that those with headaches reported every day simply have a much more severe problem that is not obvious because of the ceiling of the scale used; or that those reporting headaches every day have a degree of pain catastrophisation that prejudices the chance of a good outcome. The last of these possibilities could be examined in future work by inclusion of a score such as the Pain Catastrophizing Scale (34) at baseline.

The benefits of tONS have typically been greater in $\mathrm{CCH}$. Only case series are available, and again the outcome measures provided have not always been consistent from study to study. The most commonly reported metric is the reduction in attack frequency, and in the three largest series cluster frequency was reduced by $46 \%(n=51)(6), 50 \%(n=44)(8)$, and $58 \%(n=35)(7)$. The responder rates in these studies (percentage of patients having a $50 \%$ or greater reduction in attack frequency) were $53 \%, 59 \%$, and $67 \%$ respectively. In smaller series, outcomes have been widely different with one series reporting a 95\% reduction (5). Some studies have also reported a reduction in the intensity of residual headaches; figures range widely, from no reduction at all, up to a $50 \%$ improvement. In the larger series there were reductions in attack intensity of some $25 \%(6,8)$. All the 5 patients presented here experienced a reduction of $80 \%$ or more in attack frequency, with a mean reduction of $92 \%$. Again the number of cases in this study is too small to permit calculation of confidence intervals, but these results are encouraging. Residual headache intensity in this group fell by a mean of $42 \%$.

The outcome measures used for both types of headache have significant shortcomings. In both cases we have used a measure of frequency (cluster attack frequency or headache days) and a measure of headache intensity (NPRS). These measures are presented separately, but in reality the importance of each is dependent on the other: the intensity of residual headaches is less important if such headaches are infrequent, and the frequency of headache episodes matters less if their intensity has been dramatically reduced. A composite measure incorporating both frequency and intensity may be a better 
way to describe outcome. For example Saper et al defined responders in their migraine study as patients who saw at least a $50 \%$ reduction in headache days or at least a 3 point headache intensity reduction (25). Based on this criterion, the responder rate in the group presented here was 7 of 12 (58\%). In cluster headache, "headache load" (the product of frequency, intensity, and duration) has been proposed as an outcome measure (35) for similar reasons. We did not record headache duration and so cannot evaluate this measure for the patients in this study, but will do so in future work.

In some previous studies leads were implanted unilaterally $(2,5)$, in others bilaterally $(3,4,6,8,26)$, and in still others there was a mixture of unilateral and bilateral implantations $(7,25,27)$. Migraineurs commonly have headaches affecting both sides, although one side may be more frequently involved. Cluster headache is strictly unilateral and under ordinary circumstances always affects the same side. However this is not universally the case, and in particular side shift has been described following ONS (2, 5). In a series of unilateral implantations by Magis et al, side shift was observed in 5 of 14 cases (5), a rate of $36 \%$. In our view such a high rate justifies routine bilateral implantation at the primary procedure.

The exact anatomical site of implantation has not always been specified. Where the location has been given, it has often been at or around the level of the craniocervical junction $(25,27)$. The GON is relatively deep at this level and it is difficult to be certain that the implanted electrode is in intimate contact with it. Based on the anatomical considerations described in the introduction we have implanted leads higher up, just below the inion, similar to other studies $(6,7)$. There is also less surrounding muscle here, and we believe it is therefore less likely that stimulation will be limited by muscle activation. We note that in previous studies where lead location was well reported, the migraine studies $(25,27)$ have used the lower location while the cluster studies $(6,7)$ have used the higher implantation site, and wonder whether it is possible that this could be making a contribution to the different efficacies in the two conditions. However, this may merely be coincidence, and unfortunately 
we were unable to determine the anatomical site from the description of methods in more studies. We would urge authors in future work to make completely clear the exact anatomical electrode location in their cases.

It is impossible to perfectly blind patients in studies of paraesthesia-inducing neuromodulation treatments. In trials of tONS for $\mathrm{CM}$, investigators have gone to considerable efforts to blind as well as possible, but there are always potential confounders. The ONSTIM study compared a control group of patients who received 'preset stimulation' comprising only one minute per day of therapy at fixed levels, which they were told had been optimised for therapeutic effect, with a group who received continuous stimulation and were given a programmer with which to adjust it (25). The difference between groups was substantial (responder rates of $6 \%$ and $39 \%$ respectively), but it is difficult to exclude the possibility that the placebo effect from the continuous presence of paraesthesia was greater than that in the preset stimulation group. Providing a patient programmer to one group only is also a potential confounder because there is evidence that an element of patient choice in pain treatments enhances placebo effect (36). In the trial of Silberstein et al, all patients were provided with a programmer, but in the control group it did not communicate with the IPG (27). The same considerations as before apply with regard to the placebo effects of paraesthesia. Burst waveform stimulation is different: at clinical stimulation levels it is not perceptible to the patient, and therefore it is potentially amenable to a robustly blinded trial. We believe that such a trial of bONS is feasible and would greatly enhance the evidence base for ONS overall.

\section{Conclusion}

Paraesthesia is not necessary for ONS to be effective in $\mathrm{CM}$ and $\mathrm{CCH}$. The results in this study suggest that paraesthesia-free burst ONS can perform as well as tonic ONS or perhaps better, but larger studies 
will be required to provide a more accurate comparison of efficacy. Paraesthesia-free stimulation affords the opportunity to perform double-blind trials of ONS. 


\section{References}

1. Mosser SW, Guyuron B, Janis JE, Rohrich RJ. The anatomy of the greater occipital nerve: implications for the etiology of migraine headaches. Plast Reconstr Surg. 2004;113(2):693-7; discussion 8-700.

2. Magis D, Allena M, Bolla M, De Pasqua V, Remacle JM, Schoenen J. Occipital nerve stimulation for drug-resistant chronic cluster headache: a prospective pilot study. Lancet neurology. 2007;6(4):31421.

3. Burns B, Watkins L, Goadsby PJ. Treatment of intractable chronic cluster headache by occipital nerve stimulation in 14 patients. Neurology. 2009;72(4):341-5.

4. Muller OM, Gaul C, Katsarava Z, Sure U, Diener HC, Gasser T. [Bilateral occipital nerve stimulation for the treatment of chronic cluster headache: case series and initiation of a prospective study]. Fortschr Neurol Psychiatr. 2010;78(12):709-14.

5. Magis D, Gerardy PY, Remacle JM, Schoenen J. Sustained effectiveness of occipital nerve stimulation in drug-resistant chronic cluster headache. Headache. 2011;51(8):1191-201.

6. Miller S, Watkins L, Matharu M. Treatment of intractable chronic cluster headache by occipital nerve stimulation: a cohort of 51 patients. European journal of neurology : the official journal of the European Federation of Neurological Societies. 2017;24(2):381-90.

7. Leone M, Proietti Cecchini A, Messina G, Franzini A. Long-term occipital nerve stimulation for drug-resistant chronic cluster headache. Cephalalgia. 2017;37(8):756-63.

8. Fontaine D, Blond S, Lucas C, Regis J, Donnet A, Derrey S, et al. Occipital nerve stimulation improves the quality of life in medically-intractable chronic cluster headache: Results of an observational prospective study. Cephalalgia. 2017;37(12):1173-9.

9. Ansarinia M, Rezai A, Tepper SJ, Steiner CP, Stump J, Stanton-Hicks M, et al. Electrical stimulation of sphenopalatine ganglion for acute treatment of cluster headaches. Headache. 2010;50(7):1164-74.

10. Schoenen J, Jensen RH, Lanteri-Minet M, Lainez MJ, Gaul C, Goodman AM, et al. Stimulation of the sphenopalatine ganglion (SPG) for cluster headache treatment. Pathway $\mathrm{CH}-1$ : a randomized, shamcontrolled study. Cephalalgia. 2013;33(10):816-30.

11. Jurgens TP, Barloese M, May A, Lainez JM, Schoenen J, Gaul C, et al. Long-term effectiveness of sphenopalatine ganglion stimulation for cluster headache. Cephalalgia. 2017;37(5):423-34.

12. Franzini A, Ferroli P, Leone M, Broggi G. Stimulation of the posterior hypothalamus for treatment of chronic intractable cluster headaches: first reported series. Neurosurgery. 2003;52(5):1095-9; discussion 9-101.

13. Starr PA, Barbaro NM, Raskin NH, Ostrem JL. Chronic stimulation of the posterior hypothalamic region for cluster headache: technique and 1-year results in four patients. J Neurosurg. 2007;106(6):9991005.

14. Bartsch T, Pinsker MO, Rasche D, Kinfe T, Hertel F, Diener HC, et al. Hypothalamic deep brain stimulation for cluster headache: experience from a new multicase series. Cephalalgia. 2008;28(3):28595.

15. Kapural L, Yu C, Doust MW, Gliner BE, Vallejo R, Sitzman BT, et al. Novel 10-kHz High-frequency Therapy (HF10 Therapy) Is Superior to Traditional Low-frequency Spinal Cord Stimulation for the Treatment of Chronic Back and Leg Pain: The SENZA-RCT Randomized Controlled Trial. Anesthesiology. 2015;123(4):851-60.

16. Thomson SJ, Tavakkolizadeh M, Love-Jones S, Patel NK, Gu JW, Bains A, et al. Effects of Rate on Analgesia in Kilohertz Frequency Spinal Cord Stimulation: Results of the PROCO Randomized Controlled Trial. Neuromodulation. 2018;21(1):67-76. 
17. De Andres J, Monsalve-Dolz V, Fabregat-Cid G, Villanueva-Perez V, Harutyunyan A, AsensioSamper JM, et al. Prospective, Randomized Blind Effect-on-Outcome Study of Conventional vs HighFrequency Spinal Cord Stimulation in Patients with Pain and Disability Due to Failed Back Surgery Syndrome. Pain medicine. 2017;18(12):2401-21.

18. Deer T, Slavin KV, Amirdelfan K, North RB, Burton AW, Yearwood TL, et al. Success Using Neuromodulation With BURST (SUNBURST) Study: Results From a Prospective, Randomized Controlled Trial Using a Novel Burst Waveform. Neuromodulation. 2018;21(1):56-66.

19. Schu S, Slotty PJ, Bara G, von Knop M, Edgar D, Vesper J. A prospective, randomised, doubleblind, placebo-controlled study to examine the effectiveness of burst spinal cord stimulation patterns for the treatment of failed back surgery syndrome. Neuromodulation. 2014;17(5):443-50.

20. Courtney P, Espinet A, Mitchell B, Russo M, Muir A, Verrills P, et al. Improved Pain Relief With Burst Spinal Cord Stimulation for Two Weeks in Patients Using Tonic Stimulation: Results From a Small Clinical Study. Neuromodulation. 2015;18(5):361-6.

21. Chen YF, Bramley G, Unwin G, Hanu-Cernat D, Dretzke J, Moore D, et al. Occipital nerve stimulation for chronic migraine--a systematic review and meta-analysis. PLoS ONE. 2015;10(3):e0116786.

22. Petersen E. Improved ONS efficacy and increased pain relief in occipital neuralgia with burst stimulation: two cases. Stereotactic and functional neurosurgery. 2017;95(Suppl 1):375.

23. Headache Classification Committee of the International Headache $S$. The International Classification of Headache Disorders, 3rd edition (beta version). Cephalalgia. 2013;33(9):629-808.

24. Slotty PJ, Bara G, Kowatz L, Gendolla A, Wille C, Schu S, et al. Occipital nerve stimulation for chronic migraine: a randomized trial on subthreshold stimulation. Cephalalgia. 2015;35(1):73-8.

25. Saper JR, Dodick DW, Silberstein SD, McCarville S, Sun M, Goadsby PJ, et al. Occipital nerve stimulation for the treatment of intractable chronic migraine headache: ONSTIM feasibility study. Cephalalgia. 2011;31(3):271-85.

26. Serra G, Marchioretto F. Occipital nerve stimulation for chronic migraine: a randomized trial. Pain Physician. 2012;15(3):245-53.

27. Silberstein SD, Dodick DW, Saper J, Huh B, Slavin KV, Sharan A, et al. Safety and efficacy of peripheral nerve stimulation of the occipital nerves for the management of chronic migraine: results from a randomized, multicenter, double-blinded, controlled study. Cephalalgia. 2012;32(16):1165-79.

28. Lipton RB, Goadsby PJ, Cady RK, Aurora SK, Grosberg BM, Freitag FG, et al. PRISM study: occipital nerve stimulation for treatment-refractory migraine. Headache. 2010;50:515.

29. Rodrigo D, Acin P, Bermejo P. Occipital Nerve Stimulation for Refractory Chronic Migraine: Results of a Long-Term Prospective Study. Pain Physician. 2017;20(1):E151-E9.

30. Brewer AC, Trentman TL, Ivancic MG, Vargas BB, Rebecca AM, Zimmerman RS, et al. Long-term outcome in occipital nerve stimulation patients with medically intractable primary headache disorders. Neuromodulation. 2013;16(6):557-62; discussion 63-4.

31. Kiss ZH, Becker WJ. Occipital stimulation for chronic migraine: patient selection and complications. The Canadian journal of neurological sciences. 2012;39(6):807-12.

32. Mammis A, Sinclair GL, 3rd, Mogilner AY. Peripheral neuromodulation for headache and craniofacial pain: indications, outcomes, and complications from a single center. Clin Neurosurg. 2012;59:114-8.

33. Palmisani S, Al-Kaisy A, Arcioni R, Smith T, Negro A, Lambru G, et al. A six year retrospective review of occipital nerve stimulation practice--controversies and challenges of an emerging technique for treating refractory headache syndromes. J Headache Pain. 2013;14:67.

34. Sullivan MJL, Bishop S, Pivik J. The Pain Catastrophizing Scale: Development and validation. Psychol Assess. 1995;7:524-32. 
35. Akram H, Miller S, Lagrata S, Hyam J, Jahanshahi M, Hariz M, et al. Ventral tegmental area deep brain stimulation for refractory chronic cluster headache. Neurology. 2016;86(18):1676-82.

36. Rose JP, Geers AL, Rasinski HM, Fowler SL. Choice and placebo expectation effects in the context of pain analgesia. J Behav Med. 2012;35(4):462-70. 
Table 1: Demographics and outcomes. (HA: headache; tONS: tonic occipital nerve stimulation; bONS:

burst occipital nerve stimulation; NPRS: numeric pain rating scale).

\begin{tabular}{|c|c|c|c|c|c|c|c|c|c|c|}
\hline \multicolumn{3}{|c|}{ Demographics } & \multicolumn{2}{|c|}{ Preoperative scores } & $\begin{array}{l}\text { Previous } \\
\text { treatment }\end{array}$ & \multicolumn{5}{|c|}{ Results of bONS } \\
\hline \multicolumn{11}{|c|}{ Chronic Migraine } \\
\hline ID & Sex & Age & $\begin{array}{l}\text { HA days per } \\
\text { month }\end{array}$ & NPRS & $\begin{array}{l}\text { Previous } \\
\text { tONS }\end{array}$ & $\begin{array}{l}\text { Last follow up } \\
\text { (months) }\end{array}$ & $\begin{array}{l}\text { HA days per } \\
\text { month }\end{array}$ & $\begin{array}{c}\text { Reduction in HA } \\
\text { days }\end{array}$ & NPRS & $\begin{array}{c}\% \text { NPRS } \\
\text { reduction }\end{array}$ \\
\hline M1 & $\mathrm{F}$ & 56 & 18 & 10 & - & 18 & 12 & 6 & 6 & 40 \\
\hline $\mathrm{M} 2$ & $M$ & 40 & 30 & 10 & - & 15 & 30 & 0 & 7 & 30 \\
\hline M3 & $\mathrm{F}$ & 54 & 30 & 7 & - & 6 & 30 & 0 & 7 & 0 \\
\hline M4 & $\mathrm{F}$ & 56 & 30 & 7 & Yes & 2 & 4 & 26 & 3 & 57 \\
\hline M5 & $\mathrm{F}$ & 44 & 30 & 6 & - & 9 & 30 & 0 & 6 & 0 \\
\hline M6 & $\mathrm{F}$ & 39 & 30 & 8 & - & 9 & 30 & 0 & 6 & 25 \\
\hline M7 & $M$ & 73 & 30 & 7 & - & 8 & 6 & 24 & 7 & 0 \\
\hline M8 & $\mathrm{F}$ & 44 & 15 & 10 & - & 8 & 3 & 12 & 10 & 0 \\
\hline M9 & $\mathrm{F}$ & 31 & 30 & 9 & - & 6 & 16 & 14 & 8 & 11 \\
\hline M10 & $\mathrm{F}$ & 52 & 18 & 6 & - & 14 & 9 & 9 & 4 & 33 \\
\hline M11 & $\mathrm{F}$ & 25 & 27 & 6 & - & 13 & 18 & 9 & 6 & 0 \\
\hline \multirow[t]{2}{*}{ M12 } & M & 32 & 30 & 6 & - & 13 & 8 & 22 & 6 & 0 \\
\hline & Mean & 46 & 26.5 & 7.8 & & 10.1 & & 10.2 & & 16.4 \\
\hline \multicolumn{11}{|c|}{ Chronic Cluster Headache } \\
\hline ID & Sex & Age & $\begin{array}{l}\text { HA frequency } \\
\text { per day }\end{array}$ & NPRS & $\begin{array}{l}\text { Previous } \\
\text { tONS }\end{array}$ & $\begin{array}{l}\text { Last follow up } \\
\text { (months) }\end{array}$ & $\begin{array}{l}\text { HA frequency } \\
\text { per day }\end{array}$ & $\begin{array}{l}\% \mathrm{HA} \text { frequency } \\
\text { reduction }\end{array}$ & NPRS & $\begin{array}{c}\% \text { NPRS } \\
\text { reduction }\end{array}$ \\
\hline $\mathrm{C} 1$ & $M$ & 31 & 6 & 10 & Yes & 13 & 0 & 100 & 0 & 100 \\
\hline $\mathrm{C} 2$ & M & 41 & 2 & 10 & Yes & 20 & 0.05 & 98 & 8 & 20 \\
\hline C3 & $M$ & 35 & 5 & 10 & - & 16 & 1 & 80 & 5 & 50 \\
\hline C4 & $M$ & 60 & 20 & 10 & Yes & 6 & 4 & 80 & 6 & 40 \\
\hline \multirow[t]{2}{*}{ C5 } & $M$ & 25 & 5 & 10 & - & 3 & 0.1 & 97 & 10 & 0 \\
\hline & Mean & 38 & 7.6 & 10 & & 11.6 & & 92 & & 42 \\
\hline
\end{tabular}


Figure 1: Changes in outcome measures from baseline to last follow up. The upper two graphs relate to $\mathrm{CM}$ patients, and the lower two relate to $\mathrm{CCH}$ patients. In the left column is a measure of frequency (headache days for $\mathrm{CM}$, headache episode frequency for $\mathrm{CCH}$ ). The right column shows headache intensity for both conditions. (Note in the upper two graphs some of the horizontal lines that would have been overlapping have been separated slightly for clarity).
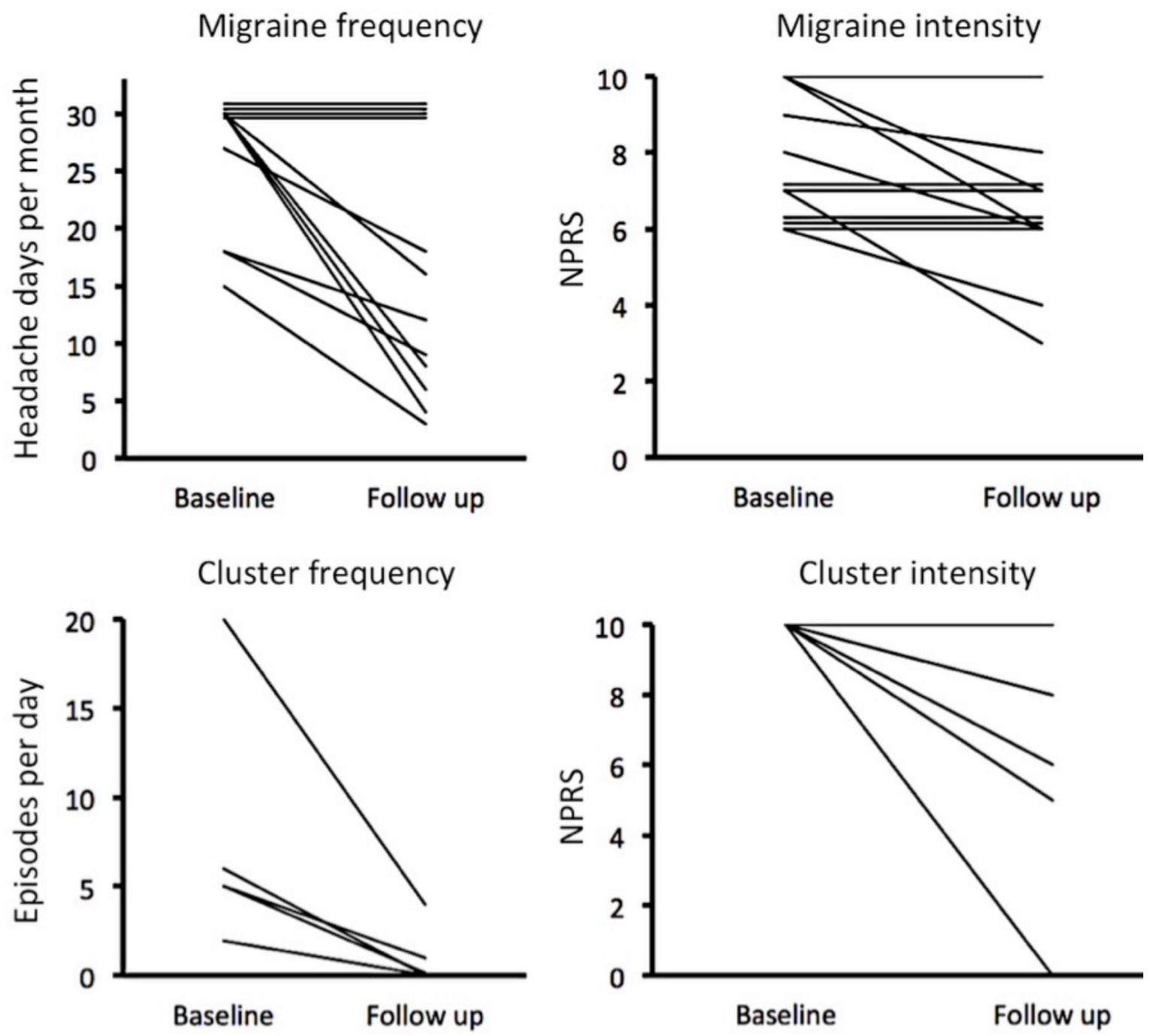